\title{
Research and practice of energy saving and consumption reducing in Data center
}

\author{
Yu Yue ${ }^{1, \text { a }}$, Kejian Cao ${ }^{2, b}$, Bin Shen, Yuan Cao ${ }^{4, d}$ and Dakun Zhang ${ }^{5, e}$ \\ ${ }^{1}$ Technical Coordination Center of National Computer Network Emergency \\ ${ }^{2}$ Technical Coordination Center of National Computer Network Emergency \\ ${ }^{3}$ China Academy of Information and Communication Technology \\ ${ }^{4}$ China Academy of Information and Communication Technology \\ ${ }^{5}$ China Academy of Information and Communication Technology

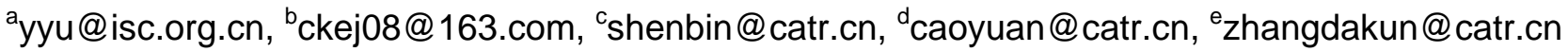

Keywords: Data Center, energy saving, PUE, engineer practice

\begin{abstract}
This paper studies the latest energy-saving technologies in domestic and international data center and focused on the important measurement of energy-saving, PUE, and finally analyzed the situation of energy saving in a real data center and proposed reform program based on engineering practice.
\end{abstract}

\section{Researches on domestic and internationl energy saving and emissions reducing technology}

\subsection{Current situation of domestic and international data center}

With the deepening of the concept of cloud computing, various large-scale data centers appear. With the rapid growth of the amount of data on Internet , the size of the data center and the number of servers and storage devices also becomes bigger and bigger. Rapid growth causes tremendous pressure to the environmental control, power and cooling, space management, etc. on data center.

According to EPA statistics, the power consumption on global data center servers has doubled from 2000 to 2005 and has increased by 75\% in 2010. Currently, a typical power consumption of small to medium data center is about 1 MW.Many large data centers, such as Google data center, need 20 megawatts of electricity to maintain its operation, which is equivalent to the power of ordinary North America 15,000 households comsume. Meanwhile, data center energy consumption has accounted for $23 \%$ of the total energy consumption of the Internet and the proportion is increasing by $12 \%$ annually. Power consumption issues in large-scale IT data center has become a domestic and international hot research field[1].

In recent years, data center are facing challenges to reduce energy consumption and cost because of climate warming and energy shortages. Data center energy efficiency receives unprecedented attention. The concept of "green data center" came into being and will be the new direction of development of data centers.

Green data center refers to the room where IT systems, machine, lighting and electric, etc. can get to maximize energy efficiency and minimize environmental impact. "Green" refers to energy saving, high reliability and rationality available on overall design and planning as well as applications of air conditioners, UPS, servers and other IT equipment and management software.

As the world's largest search engine and video service providers, Google, has been devoted itself to reducing energy consumption in its data center: Google data center has 35 million servers and consumed 1.2 billion degrees power in 2005; In 2010, Google data center has 90 million servers and power consumption only increased to 19 million degrees. Google servers accounted for $2.8 \%$ of the number of global servers, but its power consumption is less than $1 \%$ of the world. Google use a lot of energy-saving technology to reduce PUE value, such as increasing temperature standard inside the server room to 80 degrees Fahrenheit and using water cooling towers and the "evaporation" cooling and so on. PUE value in Google data center is generally not more than 1.5. 


\subsection{Domestic and international mainstream data center energy-saving technologies}

\subsubsection{Energy saving in IT room building}

IT room should be selected on the shady side of the building in order to reduce the heat generated by the sun's radiation. The main structure of the room area should be large space and large span. Building external wall is in need of a high level of thermal insulation and insulation measures on interior room, IT room floor, room ceiling should also be taken.

\subsubsection{Energy saving on IT room and cabinets layout}

IT rooms should be divided by its function and power consumption, so that the unit area of each region is relatively small. The return air with shorter distances can achieve an effective cooling and less expenses on air conditioning.

Rack with high porosity is selected and hot and cold aisles are established. Because the front of the cabinet is for air intake, rear for vents, the cabinet must be placed face to face to establish a cold aisle while back to back for hot aisle.Air conditioning cooling efficiency is strengthened to avoid hot air mixed with cold air .

High-density racks are distributed on the average throughout the room environment. If high-density rack is placed too closely, it will cause poor cooling efficiency of the cabinet easily because of hot spots.If we only strengthen the cooling effect this time, it not only causes a waste of air conditioning, but limites cabinet cooling effect.

\subsubsection{Energy saving of cooling systems}

Centralized cooling should be changed to into cooling on demand. Cooling on demand is based on the real-time needs of the heat source of each part of the heat source in the room and coolants are placed near the closest place to the heat source . Its biggest feature is the precision and quantification of cooling mode

Air flow modes contain two categories:on top and on bottom. Air flow on top can be divided into two basic types: precise air supply and inexact air supply which includes by air hood and by air ducts.

In the construction of large-scale data center cooling systems, air flow under the raised floor is often used. The entire space under the raised floor was regards as a large plenum chamber.Cold air from the air condition enters the plenum and then go through the floor outlet to cold aisle in the front of the cabinet . By adjusting ventilation area and speed of air flow on the floor before each cabinet through flow valve, the right amount of wind was supplied and the efficiency of air condition war improved . Cold air from the cold aisle is sent out into the room and cabinets and takes the heat dissipation from the equipment.Air with high temperature from the top part of room goes back to cooling treatment which can greatly save energy.

Cooling mode is divided into air-cooled and water-cooled . At present, the common way of the main data center is precise air-cooled conditioning systems. There is not enough space for outdoor condenser due to space constraints and condenser are arranged too closely resulting in poor heat dissipation and low efficiency of the air conditioning system. The Water cooling can cool exhaust hot air created by servers and other equipment in the cabinet and thus speeds up the process to improve the cooling efficiency. This water-cooled refrigeration method has been used in the large IT data center, such as Microsoft.One of the world's largest data center in Chicago data center utilize a centralized water-cooled cooling method to achieve the purpose of energy saving; Google also use by water cooling tower method to build energy efficient data center

\subsection{4 technology on using natural cooling source}

According to the using form, natural cooling source can be divided into direct and indirect natural cooling source.

Direct natural cooling source refers to the direct use of outdoor air for free cooling.The outdoor cooling air was sent to the room and cooled the indoor air. This approach requires air to be removed dust and does deal with humidity and can be used as auxiliary cooling methods.According to the local weather conditions, when the outdoor temperature is below $10{ }^{\circ} \mathrm{C}$, room cooling can use direct 
ventilation, intelligent ventilation and heat exchange, etc., to reduce or replace the traditional air conditioning cooling mode. The heat is brought from indoor to outdoor with air convection and effectively reduces the energy consumption. Currently, Tencent has been using cooling technology with natural cooling source in the green energy saving.

\subsubsection{Efficient UPS equipment}

Power Equipment -UPS system is the third energy consumer in data center and consist of input transformer, UPS and its corresponding input and output distribution cabinet. It consumes about $10 \%$ of the total power required in data center room, of which about $7 \%$ of the power comes from the power consumed by the UPS power system, about 3\% of the power comes from the UPS input system. UPS power supply are the most important part in UPS systems and includes AC/DC and DC / AC power conversion of which each has a power loss.Using high-efficient (overall efficiency) UPS power can reduce the power loss in the power conversion process and reduce the power consumption.For example, about $50,000 \mathrm{kWh} /$ year can be saved if $5 \%$ of overall efficiency is raised for an UPS with a capacity of $100 \mathrm{~kW}$

Since the new generation of the UPS has a very high efficiency, there is less chance of further increasing the efficiency of the data center by improving UPS energy effect. It is reported that APC-MGE has taken a new double conversion inverter technology which reduces $50 \%$ of the loss compared with the original design , and low power-consumed controller, the drive unit and a buffer circuit can reduce $30 \%$ of no-load loss. In the aspect of the high-voltage AC power distribution, APC-MGE eliminates isolation transformer in power distribution unit (PDU) and the resulting reduction in the loss of copper and has improved 5\% of the overall efficiency.The descrease of 4000 pounds in copper consumption in every megawatt of power and the descrease of 30,000 pounds in the weight of the PDU save the space and reduce the load on the floor.

\subsubsection{High voltage DC power supply technology}

In traditional data room power is supplied in UPS power systems or low voltage DC system (-48VDC), which are gradually being replaced with high-voltage DC power supply system. High-voltage DC power supply has the advantage of low cost, high reliability, and low energy consumption. Facebook has been using high-voltage power supply system to build the world's most energy-efficient data center by eliminating the need for centric UPS

\subsubsection{Virtualization and cloud computing technology}

The Virtualization provides a logical view rather than a physical view into the data, computing power, storage resources and other resources.With virtualization, data centers can integrate existing resources to improve the utilization of server and storage and effectively reduce the total cost of ownership (TCO) . Application of virtual resources can not only help companies improve system utilization and save hardware investment, but also run different operating systems and applications on the same server through a virtual partition. Virtualization has been seen by a business as an effective way to reduce costs by reducing the number of servers, and it can save power consumption by nearly $50 \%$.

Cloud computing is an emerging and gradually become the mainstream computing model. In this model, IT infrastructure resources, hardware resources, system resources, application resources , data resources and other IT resources are available to users on demand by way of the service on network. IT resources, composed of computing resources, are used for dynamic creation of highly virtualized resources.Cloud computing platform dynamically manage and dispatch system hardware and software resources to save computing resources and reduce maintenance costs. At present, most of the global companies use virtualization and cloud computing technology to build a green data center, such as California's Greenest Host data center which use virtualization to reduce energy loss. They will place a separate physical server to the virtual machine by virtualization to replace the original way by which one assigned to each server application or task. This can increase the efficiency of the physical server, reduce operation on data center servers, and accordingly reduced cooling requirement and the server power utilization. Under the help of virtualization, more power 
can be provided to the entire data center. After the actual run, they can save about $2000 \mathrm{kWh}$ of electricity per year

\section{Research on Power Usage Efficiency (PUE)}

\subsection{Definition on PUE}

In the year of 2007, TGG first proposed PUE (Power Usage Effectiveness) concept in the field of data center.Then, PUE is becoming a more prevalent international metrics on data center power efficiency and data center green, energy saving, environmental protection. PUE is a ratio. The closer to 1, the better the level of energy efficiency[3][4][5].PUE is calculated as:

PUE = total power consumption in data center / power consumption in IT equipment

The total power consumption is that all power to maintain the normal operation of the data center, including the power consumption of IT equipment, cooling equipment, supply and distribution systems, and other facilities. If the the office building where data center is located is used for other purposes at the same time, the power consumed in business is not included in the total power consumption in the data center. In the data center, only the power consumed by the IT equipment is considered to be "significant" power.

The actual meaning of the PUE is that in the total energy provided in the data center, how much energy is actually applied to the IT equipment. By definition, the value of PUE will rang from 1.0 to infinity. The greater the value of PUE in the data center, the more power consumed by the cooling and power supply and other supporting infrastructure. PUE's definition is simple and easy to operate. When simply measuring the total power consumption of data centers and IT equipment power consumption, we can calculate the value of PUE in the data center[2].

\subsection{Limitations of PUE}

Although PUE has a very important significance on guiding the data center to reduce energy consumption and improve energy efficiency, there are also some limitations of its own.

Undoubtedly, the use of green technology will reduce the total power consumption of the overall data center Ptotal, and power consumption of ICT equipment PIT, Assume that cooling, power supply and distribution, ICT and other green technologies to reduce the total power consumption of $\triangle$ Ptotal and simple green ICT technologies to reduce the total power consumption of $\triangle \mathrm{PIT}$, we will have the following formula:

$$
\begin{aligned}
& \mathrm{PUE}_{\text {green }}=\left(\mathrm{P}_{\text {total }}-\Delta \mathrm{P}_{\text {total }}\right) /\left(\mathrm{P}_{\text {IT }}-\Delta \mathrm{P}_{\text {IT }}\right) \\
& \mathrm{PUE}_{\text {green }}=\left(\mathrm{P}_{\text {total }}-\Delta \mathrm{P}_{\text {total }}\right) /\left(\mathrm{P}_{\text {IT }}-\Delta \mathrm{P}_{\text {IT }}\right)
\end{aligned}
$$

the difference between the PUE using a green technology and the original PUE can be obtained:

$$
\begin{aligned}
\mathrm{PUE}_{\text {green }}-\mathrm{PUE} & =\left(\mathrm{P}_{\text {total }}-\Delta \mathrm{P}_{\text {total }}\right) /\left(\mathrm{P}_{\mathrm{IT}}-\Delta \mathrm{P}_{\mathrm{IT}}\right)-\mathrm{P}_{\text {total }} / \mathrm{P}_{\mathrm{IT}} \\
& =\left(\mathrm{P}_{\text {total }} * \Delta \mathrm{P}_{\mathrm{IT}}-\mathrm{P}_{\mathrm{IT}} * \Delta \mathrm{P}_{\text {total }}\right) /\left[\left(\mathrm{P}_{\mathrm{IT}}-\Delta \mathrm{P}_{\mathrm{IT}}\right) * \mathrm{P}_{\mathrm{IT}}\right]
\end{aligned}
$$

After calculation, we can get:

$$
\begin{aligned}
& \left(\mathrm{PUE}_{\text {green }}-\mathrm{PUE}\right) *\left(\mathrm{P}_{\mathrm{IT}}-\Delta \mathrm{P}_{\mathrm{IT}}\right)=\left(\mathrm{P}_{\text {total }} * \Delta \mathrm{P}_{\mathrm{IT}}-\mathrm{P}_{\mathrm{IT}} * \Delta \mathrm{P}_{\text {total }}\right) / \mathrm{P}_{\mathrm{IT}} \\
= & \mathrm{PUE} * \Delta \mathrm{P}_{\mathrm{IT}}-\Delta \mathrm{P}_{\text {total }}
\end{aligned}
$$

We are able to determine that, PIT- $\Delta$ PIT $>0$, whereby it can be concluded: 
1) when $\Delta \mathrm{P}_{\text {total }} / \Delta \mathrm{P}_{\mathrm{IT}}>\mathrm{PUE}, \mathrm{PUE}_{\text {green }}-\mathrm{PUE}<0$, Use of green technology will bring the absolute value of PUE lower

2) when $\Delta \mathrm{P}_{\text {total }} / \Delta \mathrm{P}_{\mathrm{IT}}<\mathrm{PUE}$, $\mathrm{PUE}_{\text {green }}-\mathrm{PUE}>0$, Use of green technology will bring the absolute value of PUE higher

So, it is uncertain how the use of a variety of green technologies can impact the value of PUE. It is posssibe that the use of a greater amount of green technology increases the value of PUE. In this case , PUE show some limitations as an indicator.

\subsection{Real-time monitoring of PUE}

Through the room energy measurement and real-time monitoring system, we can achieve real-time monitoring the value of PUE. Through the communication interface in smart meter, we can achieve real-time data. Through management platform and the statistical analysis for collected data, we can provide support for communications equipment, air conditioners and other energy saving. Measurement of energy consumption is divided into three levels:

The first level is distribution power room, where electricity smart meter is installed at the end of the total power supply circuit to measure the room's total electricity consumption;

The second level is the IT room, where electricity smart meter is installed at the end of the power supply circuit to IT equipment and other electricity equipment to measure the consumption of IT equipment and other electricity equipment respectively

The third level is the array cabinet where electricity smart meter is installed inside the rack to monitor current of per rack, the total current of the array cabinet, power factor and power.

Real-time monitoring and automatic control combine all kinds of factors of racks, power distribution, air conditioning, lighting, and environment to achieve optimal operation and energy saving system throughout the room.

\section{3 energy-saving engineering practice in data center}

\subsection{Basic condition of data center}

A domestic data center room, which is a relatively new room in a northern city , was built in 2010 with a energy-saving technologies in layout of the room and cabinets, refrigeration system and efficient UPS equipment.

On energy saving in the layout of IT room and cabinets, double cabinet are opposed with glass partitions on the top and glass doors at both end and achieve the cold aisle containment.On cooling air supply, the air-cooled air-conditioning and under floor air supply are used.On UPS energy saving, the high efficient UPS from company APC are used.

Although many energy-saving measures are used as described above, the energy consumption of the entire room is still high and the value of PUE was estimated to reach 200 or more.

\subsection{Analysis of energy-saving technologies}

Under this situation, we conducted field research and analysis on the room and found the following issues:

(1) Issues on installation of air conditioning outdoor

This data center is in a high-rise office building that is not using water-cooled central air-conditioning system but outdoor unit cooling mode. With the development of business, a large number of outdoor units are installed outside. Multi-layer outdoor units are arranged and spaced too closely, resulting in poor heat dissipation and low efficiency of cooling because the air exhausted by one outdoor unit is sucked by upper outdoor unit. After measurement, the ambient temperature was about $15{ }^{\circ} \mathrm{C}$ and the the temperature around the suction outlet is up to $22.9{ }^{\circ} \mathrm{C}$.

(2) It is not tight in the cold aisle containment 
We found the closure in the cold aisle were not good and temperature in cold channel is close to the ambient temperature. The main reason is that there is a large gap between the cabinet and the equipment on both sides and after some of the equipment is off the shelf there is no installation of blank closure.The resulting air leakage is obvious in the cabinet and we can feel cold air blowed on the back of the cabinet. For these reasons, the cold aisle sealing effect is not good and cooling losses. The difference between ambient temperature and the temperature in cold aisle is only $2{ }^{\circ} \mathrm{C}$.

(3) Cabinet deployment

In one room of the data center the cold air under floor collides and leads to poor air distribution because a row with only 7 cabinets is very short although the mode of air blowing by the precision air-conditioning on both sides is used.

According to these problems, the following three recommendations about this data center optimization are proposed : (1) adjust the outdoor unit installation to try to stagger the placement or construct a cooling tower for cooling; (2) seal cold aisle of rack and install sealing plate on both sides of the cabinet and blind plate where the equipment is not installed; (3) adjust the layout of the cabinet and choose unilateral arrangement of air-conditionating when the space is suitable

\subsection{Conclusion}

Through the above practice we can find that the details of technical implementation is more important although many energy-saving technology can effectively reduce data center energy consumption, increase the value of PUE of the overall efficiency of energy. In the case of technical implementation details in place, some relatively simple energy-saving technologies can play a good role in energy-saving. Conversely, if you do not pay attention to the details of energy-saving technologies, even though you use more energy-saving technologies, it can not achieve a good energy saving effect.

It is like our green way of life that the need to start bit by bit. It deserves attention in future data center energy-saving work.

\section{References}

[1] Zhao Feng “Introduction of data center energy saving technologies” Telecom Network

Technologies, 2011

[2] cloud computing development and policy forums, "Data Center Energy Efficiency Assessment Guide", 3CPP-TR01-2012

[3] Usage and Public Reporting Guidelines for PUE/DCiE, TGG 2009.10

[4] Carbon Usage Effectiveness (CUE): A Green Grid Data Center Sustainability Metric, TGG 2010.12

[5] Water Usage Effectiveness (WUE ${ }^{\mathrm{TM}}$ ): A Green Grid Data Center Sustainability Metric, TGG 2011.3 